\title{
Mechanism of Alkyne Alkoxycarbonylation at a Pd Catalyst with P,N Hemilabile Ligands. A Density Functional Study.
}

\author{
Luke Crawford, ${ }^{[\mathrm{a}]}$ David J. Cole-Hamilton, ${ }^{[\mathrm{a}]}$ Eite Drent, ${ }^{[\mathrm{b}]}$ Michael Bühl ${ }^{\star[a]}$
}

\begin{abstract}
A detailed mechanism for alkyne alkoxycarbonylation mediated by a palladium catalyst has been characterised in detail at the B3PW91-D3/PCM level of density functional theory (including bulk solvation and dispersion corrections). This transformation, investigated via the methoxycarbonylation of propyne, involves a uniquely dual role for the $\mathrm{P}, \mathrm{N}$ hemilabile ligand acting co-catalytically as both an in-situ base and proton relay coupled with a $\mathrm{Pd}(0)$ centre, allowing for surmountable barriers (highest $\Delta \mathrm{G}^{\ddagger}$ of $22.9 \mathrm{kcal} / \mathrm{mol}$ for alcoholysis). This proton-shuffle between methanol and coordinated propyne accounts for experimental requirements (high acid concentration) and reproduces observed regioselectivities as a function of ligand structure. A simple ligand modification is proposed, which is predicted to improve catalytic turnover by three orders of magnitude.
\end{abstract}

Alkyne alkoxycarbonylation is the most atom efficient route to acrylate esters forming product in one step with $100 \%$ atom economy. ${ }^{[1-8]}$ Methoxycarbonylation of propyne yields methyl methacrylate (MMA) which is an important feedstock in industry due to its polymer poly(methyl methacrylate), more commonly known as Perspex ${ }^{[9]}$, demand for which is growing. ${ }^{[10]}$.

Drent has reported homogeneous palladium catalysts containing pyridyl phosphines - $\mathrm{P}, \mathrm{N}$ - which in the presence of acid give almost enzyme like rates of reaction $(40,000 \mathrm{~mol}(\mathrm{~mol}$ cat $\mathrm{h}^{-1}$ ) and exquisite selectivity to the branched isomer ${ }^{[2,3]}$ (Scheme 1). These conditions are also relevant to the processing of higher alkynes. ${ }^{[5,8]}$

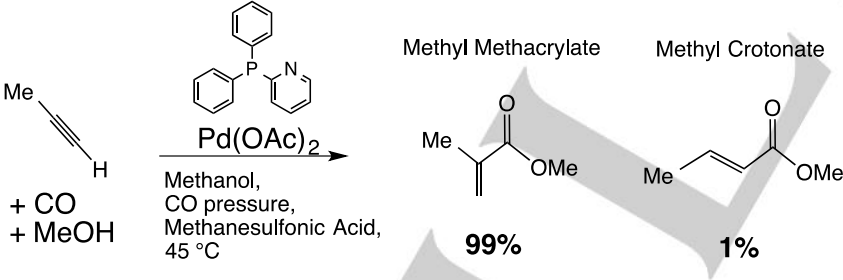

Scheme 1. Conditions for the formation of MMA involving $P, N$ ligands ${ }^{[2]}$. A high turnover of $40000 \mathrm{~mol} \mathrm{~h}^{-1}$ is observed with selectivity approximately $99 \%$ for MMA over methyl crotonate (MC).

Through methylation of the 6 position of the pyridyl moiety, the

[a] Mr. L. Crawford, Prof. D. J. Cole-Hamilton, Prof. M. Bühl, University of St. Andrews, School of Chemistry North Haugh, St. Andrews, Fife, KY16 9ST, United Kingdom E-mail: buehl@st-andrews.ac.uk

[b] Prof. E. Drent

Leiden Institute of Chemistry, Leiden University PO Box 9502, 2300 RA Leiden, The Netherlands

Supporting information for this article is given via a link at the end of the document. selectivity towards MMA over the linear product, methyl crotonate (MC), can be enhanced even further from $98.9 \%$ to $99.95 \%$ with minor changes in activity. ${ }^{[3]}$

Hemilabile $\mathrm{P}, \mathrm{N}$-type ligands with their wide range of coordination modes ${ }^{[11]}$ have attracted considerable interest in homogeneous catalysis ${ }^{[12]}$ and in order to fully elucidate the precise mode of action of 2-pyridyl diphenylphosphine (2$\left.\mathrm{PyPP}_{2}\right)$ in achieving these extraordinary results we have applied state-of-the-art density functional computations. Drent's initial work implicated a carbomethoxy mechanism with termination involving intramolecular proton transfer from a protonated pyridyl ligand ${ }^{[2]}$ whilst subsequent labeling studies by Scrivanti ${ }^{[6,7]}$ suggested that the cycle might be initiated by a proton transfer from 2-PyPPh ${ }_{2}$ onto coordinated alkyne. We have studied all pathways that have been proposed so far in the literature, as recently summarised by Cole-Hamilton and Drent. ${ }^{[1]}$ Assessing these mechanisms in detail using DFT we are able to exclude all bar one, involving proton shuffling by the pyridy groups in the initiation and termination steps, which is completely consistent with the reaction conditions and observed selectivities. Full details will be published in a forthcoming paper, here we wish to communicate the results for this preferred route and show how they enable understanding of the general alkoxycarbonylation of alkynes and can pave the way toward rational ligand design.

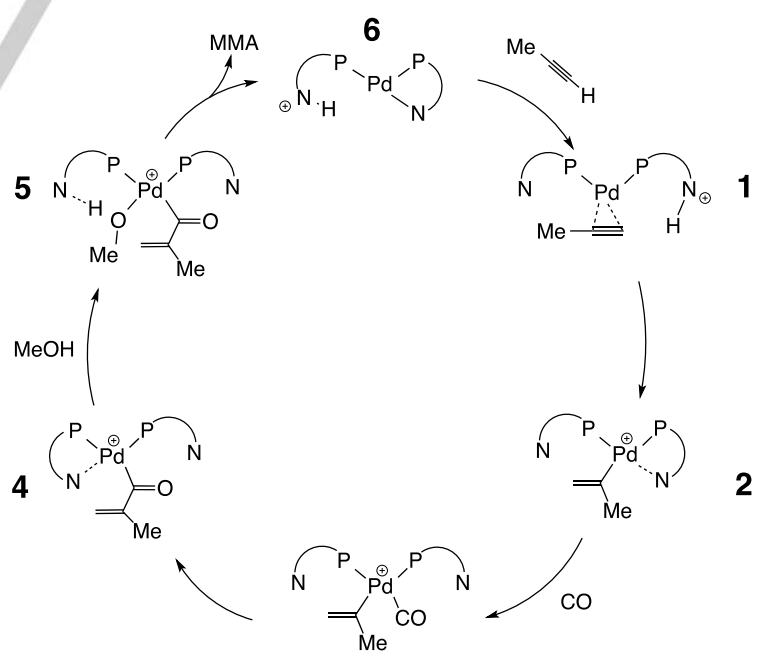

3

Scheme 2. In-situ base mechanism for catalytic MMA formation according to DFT.

This mechanism is depicted in Scheme 2. It involves two hemilabile 2- $\mathrm{PyPP}_{2}$ ligands where the dangling pyridyl moiety can act as in situ base, protonating coordinated propyne $(\mathbf{1} \rightarrow \mathbf{2})$ 
and deprotonating methanol $(5 \rightarrow 6)$. It has been proposed that the precursor complex to protonation and carbonylation may be $\left[\left(\kappa^{1}-\left(2-\mathrm{PyPPh}_{2}\right)\right)\left(\mathrm{K}^{1}-\left(2-\mathrm{PyHPPh}_{2}\right)\right) \mathrm{Pd}(\mathrm{CO})(\mathrm{HCCMe})\right]^{+}$with the protonation and migratory insertion occurring in a concerted fashion. ${ }^{[1]}$ Because CO turned out to be essentially unbound in this complex, we started the reaction from the more stable complex $\left[\left(\kappa^{1}-\left(2-\mathrm{PyPPh}_{2}\right)\right)\left(\mathrm{K}^{1}-\left(2-\mathrm{PyHPPh}_{2}\right)\right) \mathrm{Pd}(\mathrm{HCCMe})\right]^{+}(\mathbf{1})$. The free-energy profiles are shown in Schemes 3 and 4.

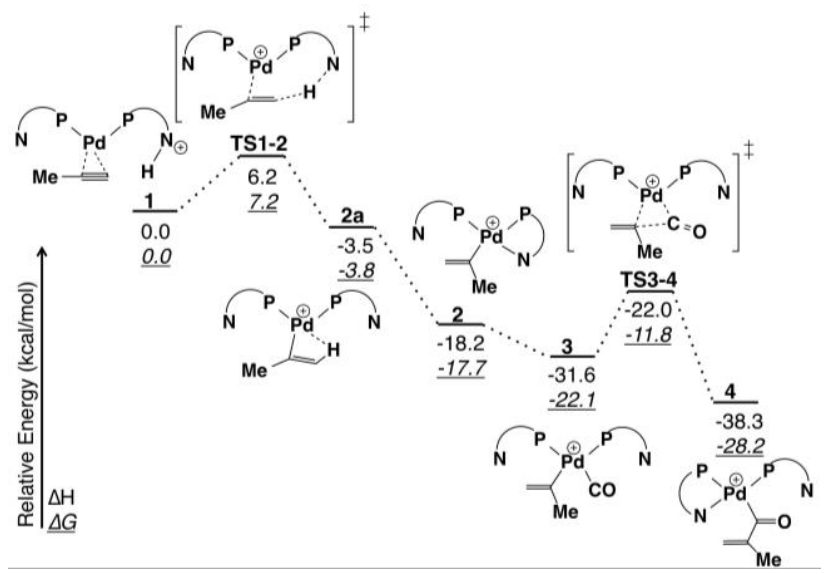

Scheme 3. Initial proton transfer and carbonylation at $\mathrm{Pd}(0)$ with two monocoordinated 2-PyPPh2 units. Energies are in $\mathrm{kcal} / \mathrm{mol}$ relative to 1 (B3PW91D3/ECP2/PCM level).

Protonation of propyne through TS1-2 is facile (activation free energy $7.2 \mathrm{kcal} / \mathrm{mol}$, Scheme 3 ) and one co-catalytic role of $\mathrm{P}, \mathrm{N}$ is established as an agent for accessible and selective proton transfer, discussed below. Subsequent CO uptake is thermodynamically driven through a large enthalpic gain that is enough to offset entropic penalties. The proceeding migratory insertion into the metal-carbon bond via TS3-4 is only 10.3 $\mathrm{kcal} / \mathrm{mol}$ uphill from the prior intermediate, and is encouraged by formation of a Pd-acyl complex. The formerly protonated pyridyl assists this process by acting as an intermittent chelate, stabilising intermediates that would otherwise be coordinatively unsaturated.

Following carbonylation a terminating alcoholysis step is necessary to yield MMA (Scheme 4). We were able to locate a transition state for nucleophilic attack at the activated Pd-acyl bond incorporating concerted deprotonation by the nitrogen of the pyridyl moiety (TS5-6). In this transition state the methanol is essentially deprotonated by the co-catalytic 2-PyPPh ${ }_{2}$ ligand and as the forming MMA product immediately dissociates away from the $\mathrm{Pd}$ centre this step is characterised as reductive elimination. Here, the P,N ligand framework demonstrates its second purpose as an in-situ base which is intrinsic in the overall high performance of alkyne functionalization by this system. Subsequent uptake of propyne to reform 1 provides the final driving force for closing the catalytic cycle. In terms of Shaik's energetic span model ${ }^{[13-15]}$ the overall barrier between the most abundant reaction intermediate (MARI, 4) and the highest energy transition state (HETS, TS5-6) is $\Delta \mathrm{G}^{\ddagger}=22.9 \mathrm{kcal} / \mathrm{mol}$, consistent with the high TOF at $45^{\circ} \mathrm{C}$. The acyl complex 4 is thus our predicted resting state (probably in equilibrium with 5), which might even prove to be detectable by ${ }^{13} \mathrm{C}$ NMR under suitable conditions.

Proton transfer through TS1-2 is followed by a series of effectively barrierless downward conversions yielding $\mathbf{3}$ after CO uptake. Thus, the insertion step is practically irreversible at turnover temperatures. This irreversibility of the regioselective transformation is essential to the high selectivities observed experimentally and arises due to the chelating mode of P,N at 3 trapping the direct product from proton transfer, preventing dynamic communication of branched and linear intermediates. To assess selectivity, we computed the path leading to the linear product through TS1-2L, which is compared to TS1-2 yielding the branched-forming intermediate in Figure 1.

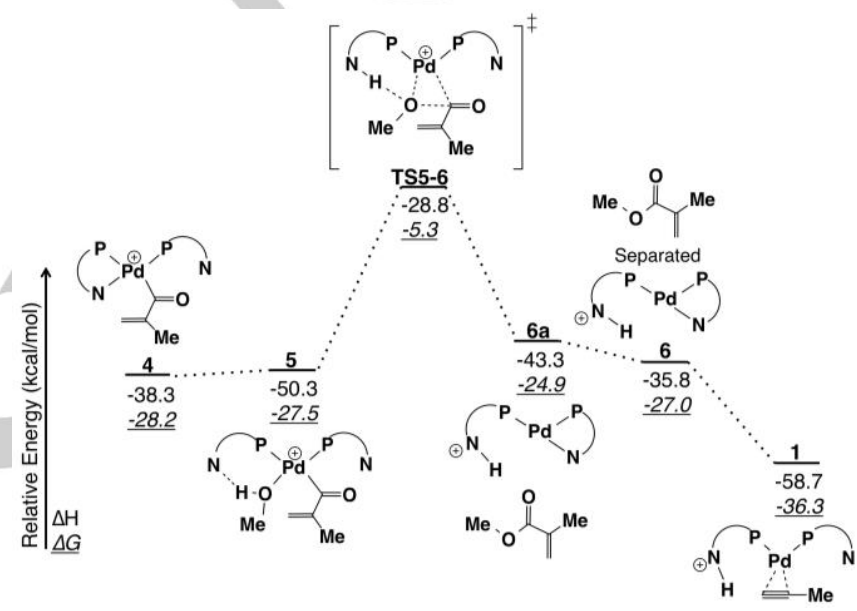

Scheme 4. Terminating methanolysis to yield MMA. Energies are in $\mathrm{kcal} / \mathrm{mo}$ relative to 1 .

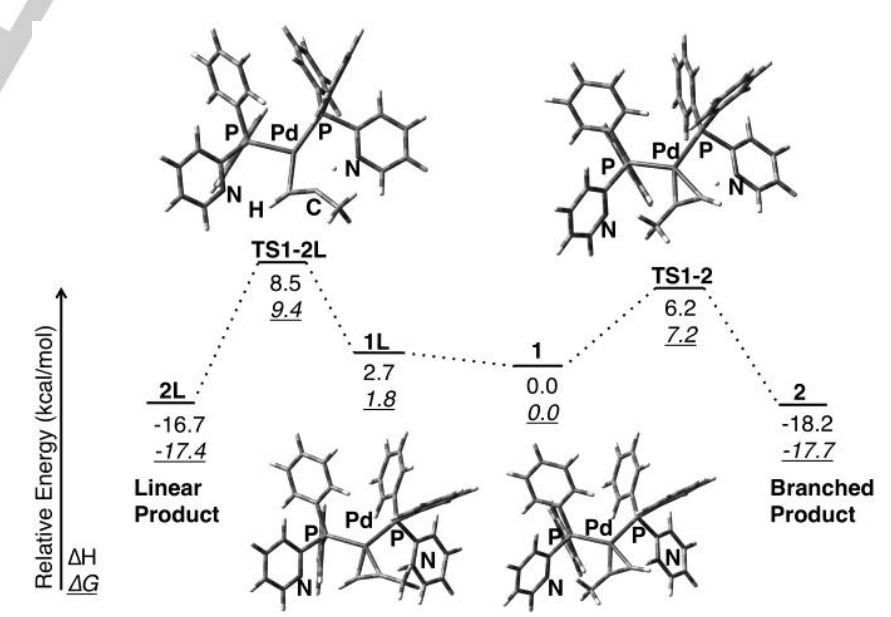

Figure 1. Pathways for formation of branched MMA (right) and linear MC (left); energies are in $\mathrm{kcal} / \mathrm{mol}$ relative to 1 .

Not only is the resultant intermediate which leads to MMA formation (2) more favourable than that leading to $\mathrm{MC}(\mathbf{2 L})$, but 
additionally the associated transition state (TS1-2) is lower in energy than that of the competing pathway, TS1-2L. $\Delta \Delta \mathrm{G}^{\ddagger}$ between these processes is computed to be $2.2 \mathrm{kcal} / \mathrm{mol}$, corresponding to a selectivity towards MMA of $98 \%$ at $25{ }^{\circ} \mathrm{C}$ and $97 \%$ at $45^{\circ} \mathrm{C}$, very close to experimental observations. The origin of this kinetic selectivity can be readily rationalised as to deliver a proton, the pyridyl moiety must move into the plane occupied by the $\pi$-coordinated propyne (Figure 1). For TS1-2 this occurs with no pronounced steric clashing whereas for the MC-forming TS1-2L, this movement introduces an unfavourable interaction between the aromatic ring and the methyl group of propyne, ultimately leading to a higher barrier.

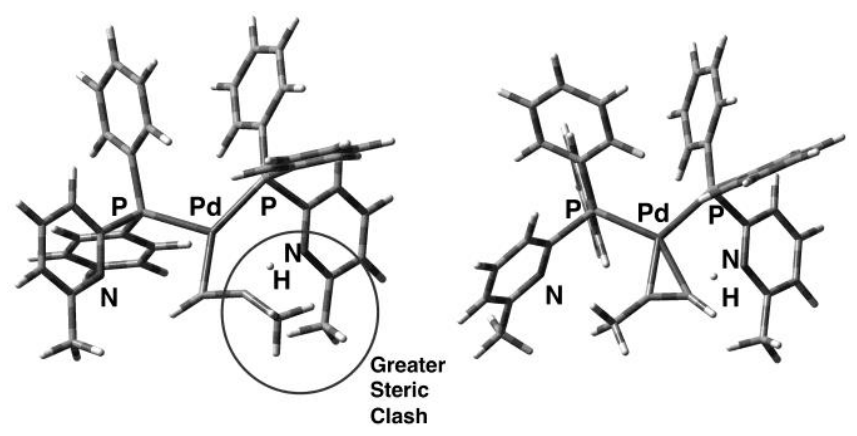

Figure 2. Selectivity determining transition states for the 6-methyl analogues of TS1-2 (right) and T1-2L (left). Note the enhanced steric clash in the MCforming transition state.

At our applied level of DFT 6-methylation leads to an increase in selectivity towards MMA over $M C$, with $\Delta \Delta G^{\ddagger}$ rising from $2.2 \mathrm{kcal} / \mathrm{mol}$ to $4.1 \mathrm{kcal} / \mathrm{mol}$ (due to a subtle ligand effect illustrated in Figure 2). The latter value is in excellent agreement with the $99.95 \%$ regioselectivity observed experimentally with 2 (6-Me) $\mathrm{PyPPh}_{2}$ ligands.

Our results explain why the P,N system is strikingly different from other ligands used in catalytic carbonylation at $\mathrm{Pd}$. Typically, these involve bidentate diphosphine backbones and give high selectivities towards linear (alkoxy)carbonylation products $^{[16-19]}$. By contrast, 2-PyPPh ${ }_{2}$ and congeners undergo an alternative "Pd - $\mathrm{H}$ " cycle and it is the unique mechanistic inclusion of the pyridyl moiety, which actively participates in two distinct transformations - controlling both regioselectivity and overall TOF - that enables this framework to conduct orthogonal selectivity and strongly favour branched products over linear. This dual role of the ligand, anticipated by Drent ${ }^{[2]}$ and confirmed by our results, has also been invoked in transfer hydrogenation reactions with other $\mathrm{N}$-donor ligands ${ }^{[20,21]}$.

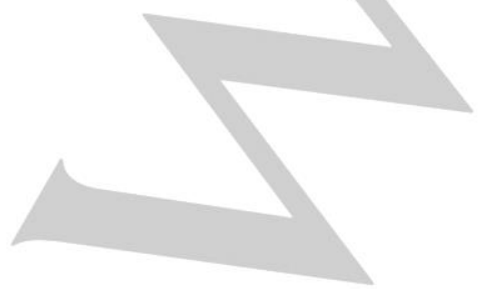

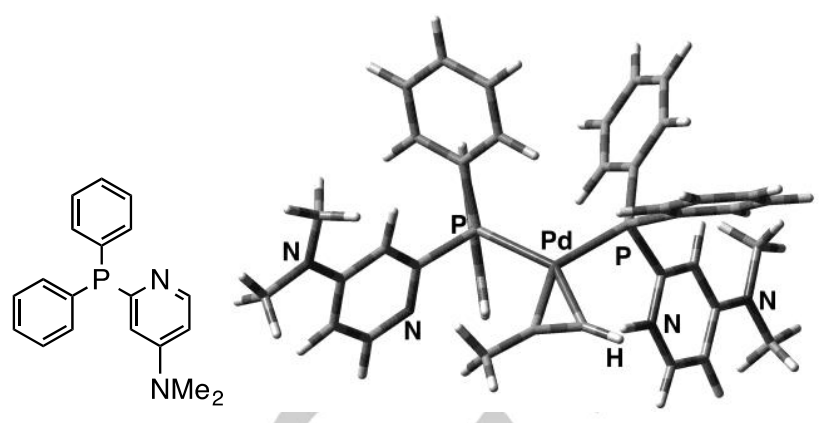

Figure 3. Left: Predicted higher activity ligand framework incorporating a dimethylamino group at the para position of the pyridyl moiety; Right: Analogue of complex 1 with two of these ligands.

How can the insights from our DFT results be used to inform on the design of improved catalysts? From the key role of the pyridyl arm as an in-situ base one may expect the basicity of this moiety to be of prime importance. Aiming to increase this, and thus improve the catalyst, we have changed the hydrogen atom at the para position (relative to the pyridyl nitrogen) into a dimethylamino group and recomputed the entire pathway (see Figure 3 for a plot of the starting point, modified complex 1). Whilst this functionalisation increases the proton transfer barrier via TS1-2 (cf. Scheme 3) from $7.2 \mathrm{kcal} / \mathrm{mol}$ to $12.3 \mathrm{kcal} / \mathrm{mol}$, the crucial methanolysis process via TS5-6 (cf. Scheme 4) is expedited with a predicted cost of $19.3 \mathrm{kcal} / \mathrm{mol}$, which is 3.6 $\mathrm{kcal} / \mathrm{mol}$ lower than that of the parent catalyst. Such a decrease in barrier increases the predicted TOF by almost three orders of magnitude. This first encouraging result illustrates how rational design will be focused upon balancing electronics such that TS1-2 remains accessible as TS5-6 is lowered.

We note that this ligand system with the 4dimethylaminopyridyl moiety is actually known and has already been used in catalytic studies ${ }^{[2,23]}$ and thus could offer a readily accessible means for testing our predictions.

In conclusion, we have used DFT to characterise a viable, low-energy pathway for catalytic alkoxycarbonylation of alkynes at a homogeneous $\mathrm{Pd}$ catalyst with $\mathrm{P}, \mathrm{N}$-type ligands, using the production of MMA to exemplify this mechanism. This stepwise $\mathrm{Pd}(0)$ route with essential co-catalytic hemilabile $\mathrm{PyPPh}_{2}$ ligands involves a regioselective irreversible protonation of coordinated propyne, subsequent $\mathrm{CO}$ insertion and in-situ base-assisted alcoholysis. Accounting for the requirement of excess ligand and acid (to keep two ligands coordinated and to have a dangling pyridyl arm protonated) and correctly reproducing observed selectivities as a function of ligand bulk, this mechanism is fully consistent with all available experimental data. We trust that our detailed computational insights, which account for reactivities and selectivities at an atomistic level, will spur the design and development of new generations of more active, and more selective catalyst systems for this important feedstock molecule. We have already taken the first steps along this line, showing how a testable ligand modification could lead to a significant speedup, thus presenting the first targeted in silico ligand design for this system. 


\section{Experimental Section}

Energies have been computed at the B3PW91 ${ }^{[24-26]}-\mathrm{D} 3^{[27]} / \mathrm{SDD}(\mathrm{Pd}) / 6$ $311+\mathrm{G}^{\star *} / \mathrm{PCM}^{[28]}(\mathrm{MeOH})$ level on B3PW91/SDD $(\mathrm{Pd}) / 6-31 \mathrm{G}^{* *}$ geometries and corrected for enthalpies and free energies using harmonic vibrational frequencies at the latter level. For details and references see Supporting Information.

\section{Acknowledgements}

The authors would like to thank the School of Chemistry and EaStCHEM for support and for access to a computing facility maintained by Herbert Früchtl.

Keywords: alkynes $\cdot$ density functional calculations $•$ homogeneous catalysis $\cdot$ palladium $\bullet$ reaction mechanisms

[1] D. J. Cole-Hamilton, E. Drent, in Appl. Homog. Catal. with Organomet. Complexes, Wiley VCH, Weinheim, 2014.

[2] E. Drent, P. Arnoldy, P. H. M. Budzelaar, J. Organomet. Chem. 1993, 455, 247-253.

[3] E. Drent, P. Arnoldy, P. H. M. Budzelaar, J. Organomet. Chem. 1994, 475, 57-63.

[4] A. Dervisi, P. G. Edwards, P. D. Newman, R. P. Tooze, S. J. Coles, M. B. Hursthouse, J. Chem. Soc. Dalt. Trans. 1999, 3, 1113-1120.

[5] A. Dervisi, P. G. Edwards, P. D. Newman, R. P. Tooze, J. Chem. Soc. Dalt. Trans. 2000, 523-528.

[6] A. Scrivanti, V. Beghetto, E. Campagna, M. Zanato, U. Matteoli, Organometallics 1998, 17, 630-635.

[7] A. Scrivanti, M. Bertoldini, V. Beghetto, U. Matteoli, A. Venzo, J. Organomet. Chem. 2009, 694, 131-136.
[8] C. S. Consorti, G. Ebeling, J. Dupont, Tetrahedron Lett. 2002, 43, 753-755.

[9] B. Harris, Ingenia 2010, 18-23.

[10] M. Moukwa, Chem. World 2010, 50-52.

[11] J. Liu, C. Jacob, K. J. Sheridan, F. Al-Mosule, B. T. Heaton, J. A. Iggo, M. Matthews, J. Pelletier, R. Whyman, J. F. Bickley, et al., Dalton Trans. 2010, 39, 7921-35.

[12] P. Kumar, A. K. Singh, M. Yadav, P. Li, S. K. Singh, Q. Xu, D. S. Pandey, Inorganica Chim. Acta 2011, 368, 124-131.

[13] S. Kozuch, S. Shaik, J. Am. Chem. Soc. 2006, 128, 3355-3365.

[14] S. Kozuch, S. E. Lee, S. Shaik, Organometallics 2009, 28, 13031308.

[15] S. Kozuch, S. Shaik, Acc. Chem. Res. 2011, 44, 101-10.

[16] J. Tijani, R. Suleiman, B. El Ali, Appl. Organomet. Chem. 2008, 22 , 553-559.

[17] R. Suleiman, J. Tijani, B. El Ali, Appl. Organomet. Chem. 2009, 24, 38-46.

[18] A. A. Núñez Magro, L.-M. Robb, P. J. Pogorzelec, A. M. Z. Slawin, G. R. Eastham, D. J. Cole-Hamilton, Chem. Sci. 2010, 1, 723.

[19] C. Jimenez-Rodriguez, G. R. Eastham, D. J. Cole-Hamilton, Dalt Trans. 2005, 2, 1826-1830

[20] R. Noyori, M. Yamakawa, S. Hashiguchi, J. Org. Chem. 2001, 66, 7931-44.

[21] D. A. Alonso, P. Brandt, S. J. M. Nordin, P. G. Andersson, J. Am Chem. Soc. 1999, 121, 9580-9588.

[22] R. García-Álvarez, S. E. García-Garrido, J. Díez, P. Crochet, V. Cadierno, Eur. J. Inorg. Chem. 2012, 2012, 4218-4230.

[23] D. M. Zink, M. Bächle, T. Baumann, M. Nieger, M. Kühn, C. Wang, W. Klopper, U. Monkowius, T. Hofbeck, H. Yersin, et al., Inorg. Chem. 2013, 52, 2292-305

[24] A. D. Becke, J. Chem. Phys. 1996, 104, 1040-46.

[25] J. P. Perdew, J. A. Chevary, V. S. H, K. A. Jackson, M. R. Pederson, D. J. Singh, C. Fiolhais, Phys. Rev. B. 1992, 46, 6671-6687.

[26] J. P. Perdew, K. Burke, Y. Wang, Phys. Rev. B. 1996, 54, 533-539.

[27] T. Risthaus, S. Grimme, J. Chem. Theory Comput. 2013, 9, 15801591.

[28] J. Tomasi, B. Mennucci, E. Cancés, J. Mol. Struct. Theochem 1999 $464,211-226$. 
Entry for the Table of Contents (Please choose one layout)

Layout 2:

\section{COMMUNICATION}

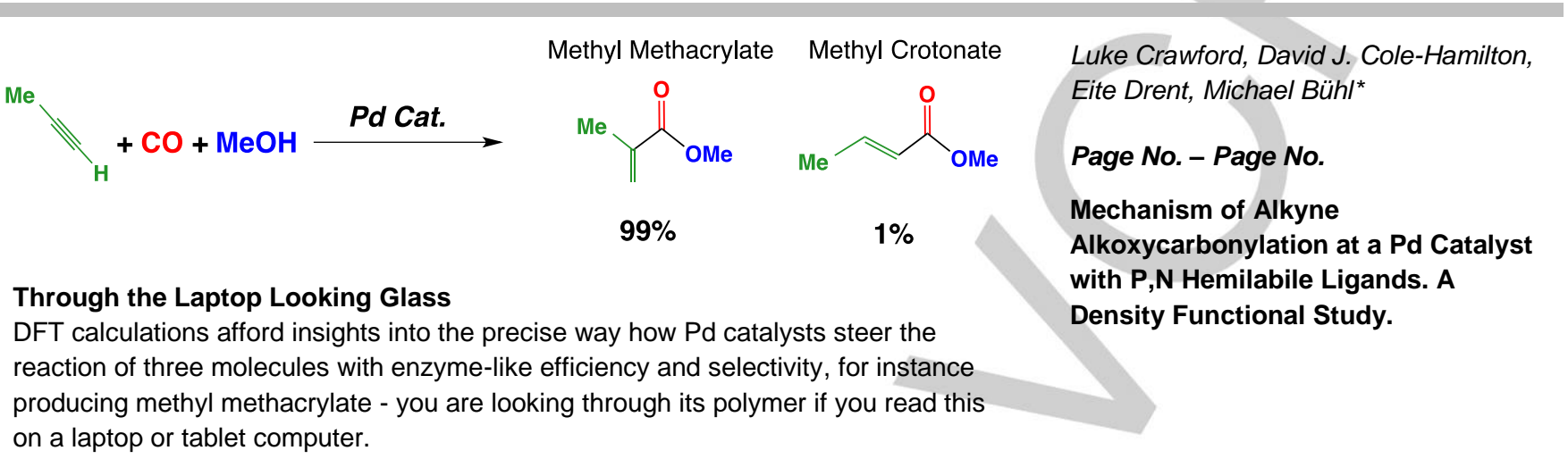
on a laptop or tablet computer. 